\title{
ESTABILIDAD EN CONDICIONES ESTÁTICAS Y PROBABILIDAD DE OCURRENCIA DE DESLIZAMIENTOS EN CONDICIONES PSEUDOESTÁTICAS DE LAS LADERAS DEL BAJO LOS ANONOS, SAN RAFAEL, ESCAZÚ, SAN JOSÉ. Rolando Mora Chinchilla
}

\section{Resumen}

Se ha realizado un reconocimiento geológico, geomorfológico y geotécnico del Bajo Los Anonos, con el objetivo de establecer todos los parámetros y sus distribuciones de funciones de probabilidades, para así proceder a calcular el factor de seguridad en condiciones estáticas y la probabilidad de ocurrencia de deslizamientos en caso de actividad sísmica. En términos generales, se puede concluir que poco más de un sexto del área del Bajo Los Anonos se puede considerar segura para la construcción de infraestructura habitacional.

Palabras claves: estabilidad de laderas, amenaza, deslizamientos, gestión del riesgo, geotecnia.

\section{Abstract}

A geological, geomorphological and geotechnical recognition has been done of Bajo Los Anonos, aiming to establish all the parameters and their distributions of probability functions, in order to proceed to calculate the safety factor in static conditions and the probability of occurrence of slides in case of seismic activity. In general terms, it can be concluded that little more than one sixth of the area at BajoAnonos is consider safe for the construction of housing infrastructure.

Keywords: slope stability, hazard, slip, risk management, geotechnics.

Recibido: 02 de diciembre del 2007 - Aprobado: 12 de abril del 2010

\section{INTRODUCCIÓN}

La comunidad de Bajo Los Anonos se localiza $1,5 \mathrm{~km}$ al oeste de la esquina suroeste del Parque Metropolitano La Sabana, en el Distrito San Rafael, Cantón de Escazú, Provincia de San José (Figura 1), entre las coordenadas (212 500$213000) \mathrm{N}$ y (522 600-523 100) E.

En este trabajo se realiza una descripción de la situación geológica y geomorfológica del sitio donde se localiza el Bajo Los Anonos y se analiza la probabilidad de ocurrencia de deslizamientos.

Se pretende que el resultado de esta investigación sirva como herramienta para la toma de decisiones de la Municipalidad de Escazú, en lo que respecta a la gestión preventiva del riesgo en la comunidad del Bajo Los Anonos.

\section{GEOLOGÍA}

Las siguientes unidades geológicas afloran en el Bajo Los Anonos (Figura 2):

Formación Tiribí (Qv-t): Depósitos de ignimbritas grises con fiames de obsidiana y pómez.

Lahares y cenizas (Qvl): Materiales procedentes de los volcanes de la Cordillera Volcánica Central, compuestos por avalanchas de lodo y rocas, y cenizas volcánicas.

Depósitos aluviales (Qal): Depósitos de centimétricos a métricos de bloques de lavas andesíticas, subredondeados y asociados al transporte fluvial del río Tiribí. 


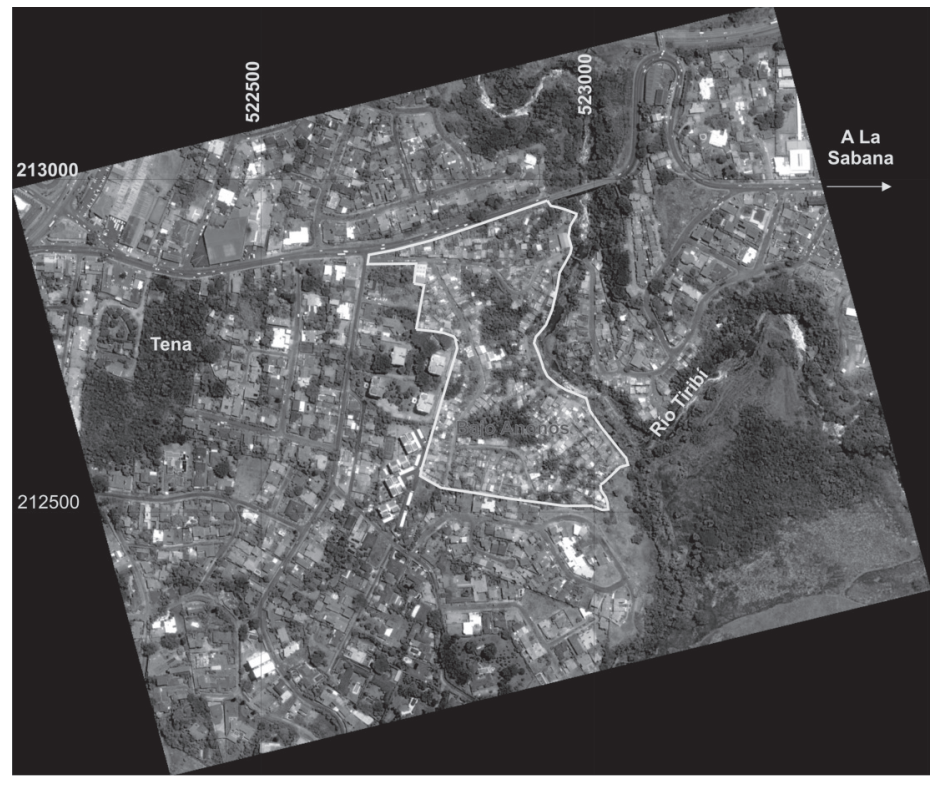

Figura 1. Localización del área de estudio. Fuente: (El autor).

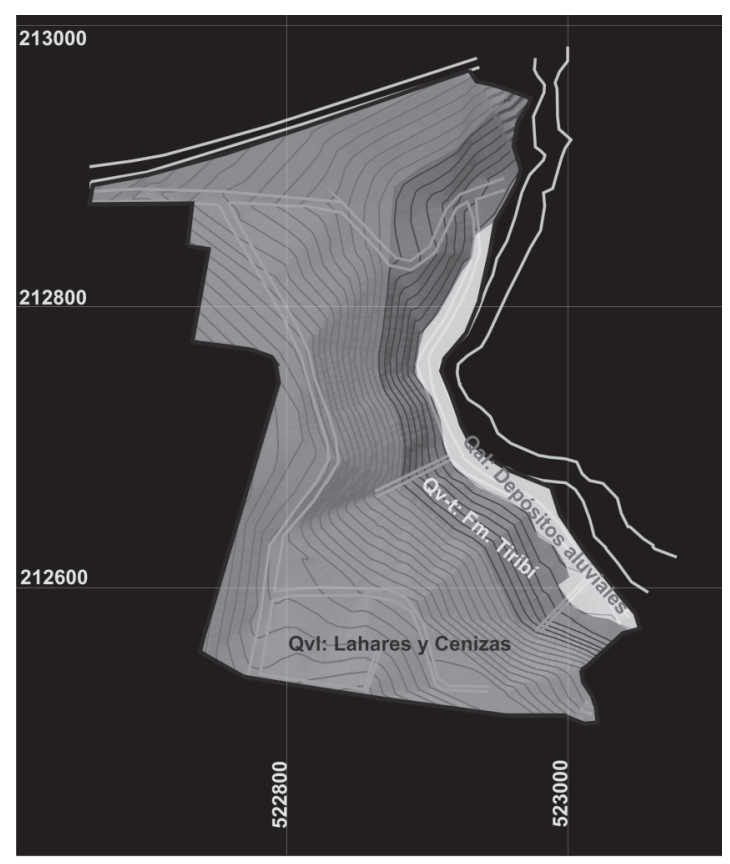

Figura 2. Mapa geológico del Bajo Los Anonos, Escazú, San José.

Fuente: (El autor). 


\section{GEOMORFOLOGÍA}

Las siguientes son las Formas/Unidades Geomorfológicas identificadas en el Bajo Los Anonos (Figura 3):

F-01 Terraza fluvial: Topografía de plana a levemente inclinada, comprendida entre el lecho menor del río Tiribí y la base del cañón del mismo río. Este sector se encuentra sujeto a inundaciones durante eventos extremos.

F-02 Cañón fluvial: Topografía de pendientes fuertes a muy fuertes, desarrollada a partir de procesos de erosión fluvial. Esta forma del terreno es muy susceptible a desarrollar eventos de deslizamiento y flujos de lodo y rocas.

V-01 Campos de lahares y cenizas: Topografía de suave a moderada, levemente disectada, con desarrollo importante de suelos. Sector susceptible a desarrollar deslizamientos, si se realizan cortes y se dejan sin soporte.

\section{GEOTECNIA}

A partir de cuatro muestreos de campo, doce ensayos de laboratorio y cuatro de campo, se han establecido las Unidades Geotécnicas del Bajo Los Anonos (Figura 4):

Aluvión grueso: Bloques de roca que componen la terraza fluvial, se encuentran en la parte plana cercana al río Tiribí. Es un depósito soportado por bloques y no presenta un contenido de matriz significativo.

Afloramiento rocoso: Macizo rocoso fracturado, compuesto por ignimbritas de la Formación Tiribí. Mediante estudios geomecánicos se ha establecido que la cohesión del macizo oscila entre $200 \mathrm{kPa}$ y $300 \mathrm{kPa}$, y que el ángulo de fricción del macizo lo hace entre $25^{\circ}$ y $35^{\circ}$ (Mora, 1999).

Suelos volcánicos: Limos elásticos, limos elásticos con arena y limos elásticos arenosos (MH), de plasticidad alta. Para establecer las

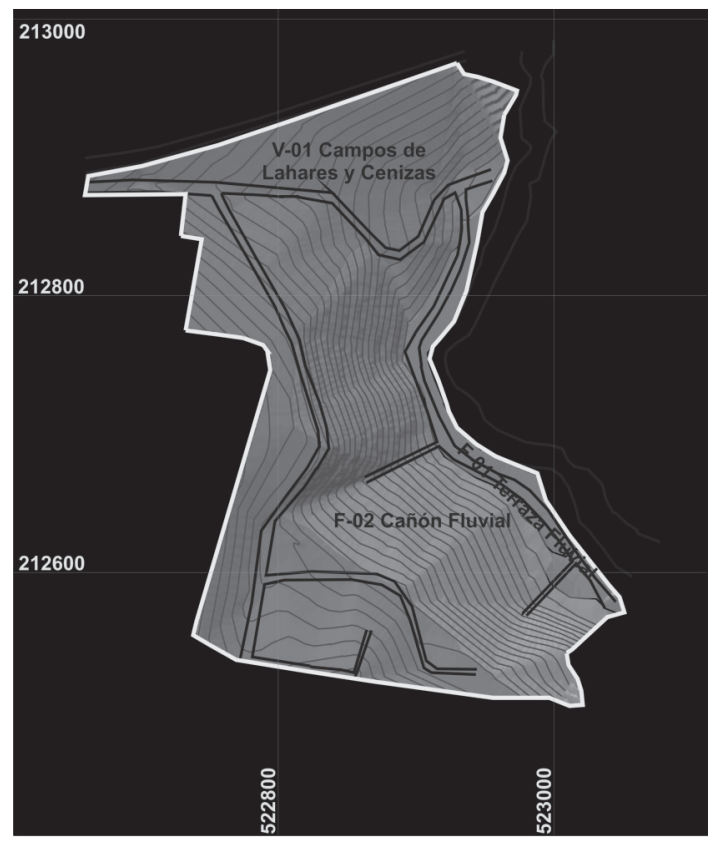

Figura 3. Mapa geomorfológico del Bajo Los Anonos, Escazú, San José. Fuente: (El autor). 


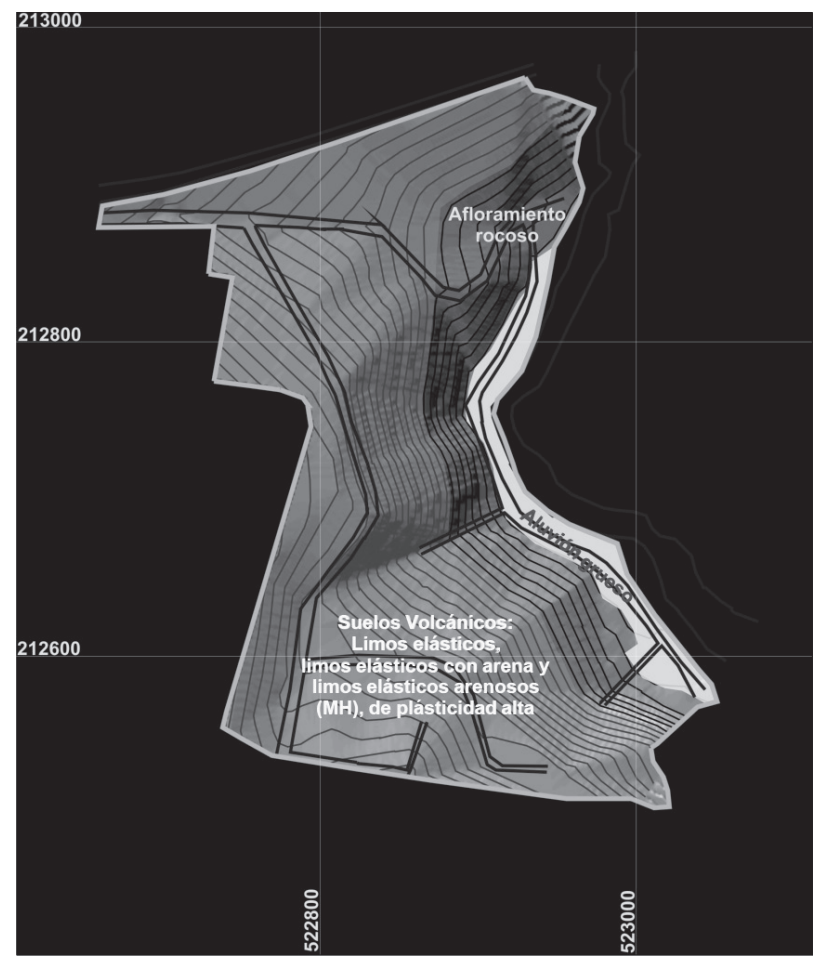

Figura 4. Mapa Geotécnico del Bajo Los Anonos, Escazú, San José.

Fuente: (El autor).

propiedades físicas y mecánicas de esta Unidad Geotécnica, se realizaron sondeos y muestreos en cuatro diferentes localidades del área de estudio. Un resumen de los resultados de los ensayos se presenta en el Cuadro 1.

\section{ANÁLISIS DE ESTABILIDAD Y CÁLCULO DE LA PROBABILIDAD DE OCURRENCIA DE DESLIZAMIENTOS}

Para analizar la estabilidad en condiciones estáticas y calcular la probabilidad de ocurrencia de deslizamientos en condiciones pseudoestáticas, se ha utilizado el programa de cómputo PISA (Probabilistic Infinite Slope Analysis) (Haneberg, 2005), el cual ejecuta cálculos de estabilidad de laderas probabilísticos, en condiciones estáticas y dinámicas, a partir de modelos de elevación digitales del terreno. Se basa en la formulación de primer orden segundo momento (FOSM) de la ecuación del talud infinito, utilizada por los programas LISA y DLISA del U.S. Forest Service, y por lo tanto, puede incluir los efectos de la resistencia de las raíces de los árboles y la sobrecarga de los mismos (Haneberg, 2005).

PISA es un excelente complemento para los inventarios de deslizamientos basados en fotointerpretación y trabajo de campo y puede ser usado para evaluar los efectos potenciales de la deforestación u otras actividades a escala de cuenca. También puede ser empleado para evaluar el potencial de deslizamiento a lo largo de vías de comunicación u otras obras lineales, así como en la planificación de uso del terreno y proyectos de zonificación (Haneberg, 2005).

\subsection{Parámetros de entrada}

El modelo de elevación digital se ha generado a partir de las curvas de nivel proporcionadas por la Municipalidad de Escazú, las cuales representan las elevaciones topográficas con intervalos de 2,0 m. El tamaño de la rejilla utilizada para la 
Cuadro 1. Propiedades físicas y mecánicas de los suelos volcánicos del Bajo Los Anonos, Escazú, San José.

Propiedad

Gravedad específica
Contenido de humedad $(\%)$
Peso unitario húmedo $\left(\mathrm{kN} / \mathrm{m}^{3}\right)$
Peso unitario seco $\left(\mathrm{kN} / \mathrm{m}^{3}\right)$
Peso unitario saturado $\left(\mathrm{kN} / \mathrm{m}^{3}\right)$
Peso unitario de los sólidos $\left(\mathrm{kN} / \mathrm{m}^{3}\right)$
Relación de vacíos
Porosidad (\%)
Grado de saturación (\%)
Porcentaje de finos $(\%)$
Porcentaje de arena (\%)
Porcentaje de grava (\%)
Límite líquido (\%)
Límite plástico (\%)
Índice de plasticidad (\%)
Cohesión drenada $(\mathrm{kPa})$
Ángulo de fricción drenado $\left({ }^{\circ}\right)$
Clasificación SUCS

\section{Resultados}

$2,41-2,51$

$29,9-43,0$

$14,7-16,3$

$10,7-12,6$

$16,1-17,2$

$23,6-24,6$

0,90-1,29

$47,3-56,4$

$73,4-95,4$

64,3-95,2

4,8-31,9

$1,6-3,8$

$50,9-68,4$

$35,0-42,8$

$15,9-25,6$

$4-8$

27-36

$\mathrm{MH}$ confección del modelo y para la realización de todos los cálculos posteriores es de $1 \mathrm{~m} \mathrm{x} 1 \mathrm{~m}$.

Los parámetros de resistencia al corte de los suelos volcánicos se obtuvieron mediante la realización de ensayos de corte directo en muestras inalteradas. El ángulo de fricción interna de los suelos presenta una distribución uniforme y oscila entre $27^{\circ}$ y $36^{\circ}$. La cohesión de los suelos se considera como de distribución triangular y sus valores oscilan de 4 $000 \mathrm{~Pa}$ a $10000 \mathrm{~Pa}$, con un pico de $8000 \mathrm{~Pa}$.

Según la visita de campo, se ha establecido que el espesor deslizable puede oscilar de $1,1 \mathrm{~m}$ a 3,1 $\mathrm{m}$ y se le ha asignado una distribución uniforme.

Debido a que no se da la presencia de niveles de agua en los suelos volcánicos, el coeficiente de presión de poros no se ha tomado en consideración, pero el efecto de pérdida de resistencia por humedecimiento se ha incluido al realizar los ensayos de corte directo en condiciones de saturación total.

El peso unitario saturado cuenta con una distribución triangular y oscila de $16100 \mathrm{~N} / \mathrm{m}^{3}$ a
$17200 \mathrm{~N} / \mathrm{m}^{3}$, con un pico de $16300 \mathrm{~N} / \mathrm{m}^{3}$. El peso unitario húmedo también tiene una distribución triangular y oscila de $14700 \mathrm{~N} / \mathrm{m}^{3}$ a $16400 \mathrm{~N} / \mathrm{m}^{3}$, con un pico de $16300 \mathrm{~N} / \mathrm{m}^{3}$.

Para calcular la probabilidad de ocurrencia de deslizamientos en condiciones pseudoestáticas, se ha utilizado un coeficiente de aceleración sísmica de $0,2 \mathrm{~g}$.

\subsection{Resultados del análisis de estabilidad en condiciones estáticas}

Este análisis se realiza calculando el factor de seguridad para cada pixel del mapa y reclasificando los resultados de acuerdo con el Cuadro 2. El mapa final (Figura 5) incluye la incorporación de procesos relacionados con la ocurrencia de flujos de lodo y rocas, así como de inundaciones.

En la Figura 6 se presenta la distribución porcentual de las diferentes clases del mapa de 
Cuadro 2. Conceptos utilizados para la reclasificación del mapa de estabilidad en condiciones estáticas, Bajo Los Anonos, Escazú, San José.

\section{Clase}

1

Laderas estables

5

6

7 rocas

Sector inundable

\section{Nombre}

Laderas moderadamente estables

Laderas inestables

Laderas muy inestables

Sector afectable por flujos de lodo y

Sector afectable por flujos de lodo y rocas e inundaciones

\section{Factor de} seguridad

Mayor de 1,4

De 1,2 a 1,4

De 1,0 a 1,2

Menor de 1,0

Se deben realizar medidas correctivas menores para asegurar la estabilidad del sector.

Potencial alto de deslizamientos, se deben implementar medidas correctivas mayores para asegurar la estabilidad del sector.

Potencial muy alto de deslizamientos, se deben implementar medidas correctivas mayores para asegurar la estabilidad del sector.

Posibilidad de ocurrencia de flujos y avalanchas de lodo y rocas, disparadas por deslizamientos en la sección superior de la ladera.

Posibilidad de ocurrencia de inundaciones durante eventos extremos del río Tiribí.

Posibilidad de conjugación de eventos de flujos de lodo y rocas e inundaciones.

Fuente: (El autor).

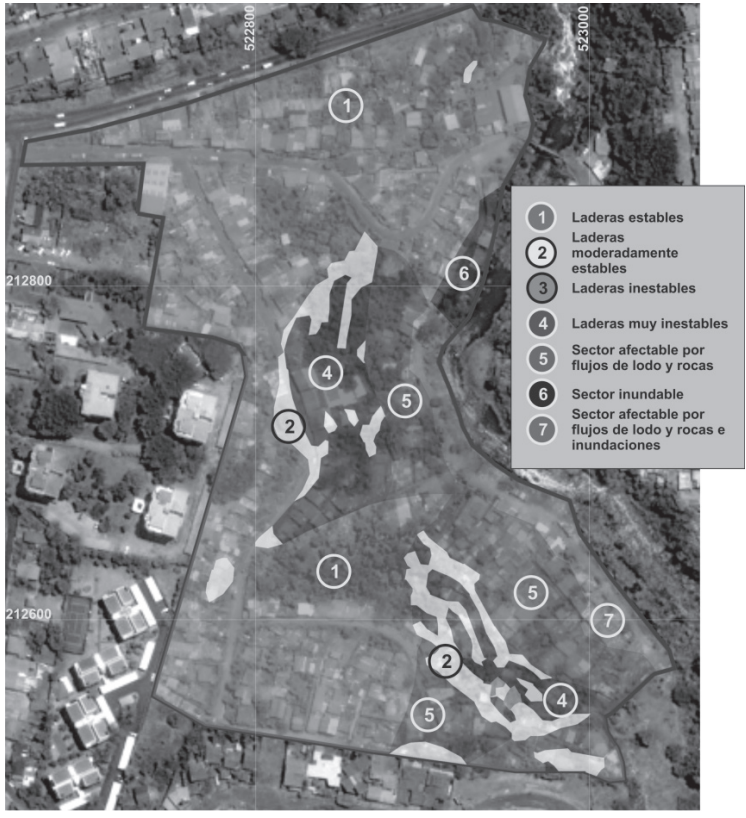

Figura 5. Mapa de estabilidad en condiciones estáticas y procesos geomorfológicos conexos, del Bajo Los Anonos, Escazú, San José.

Fuente: (El autor). 
Cuadro 3. Conceptos utilizados para la reclasificación del mapa de estabilidad en condiciones pseudoestáticas, Bajo Los Anonos, Escazú, San José.

\begin{tabular}{|c|c|c|}
\hline Clase & $\begin{array}{c}\text { Probabilidad de ocurrencia } \\
\text { de deslizamientos }\end{array}$ & $\begin{array}{l}\text { Probabilidad } \\
\text { en términos } \\
\text { porcentuales }\end{array}$ \\
\hline 1 & Baja & $<10 \%$ \\
\hline 2 & Moderada & $10 \%-20 \%$ \\
\hline 3 & Alta & $20 \%-50 \%$ \\
\hline 4 & Muy alta & $50 \%-100 \%$ \\
\hline 5 & $\begin{array}{l}\text { Sector afectable por flujos de } \\
\text { lodo y rocas }\end{array}$ & \\
\hline 6 & $\begin{array}{l}\text { Sector afectable por flujos de } \\
\text { lodo y rocas e inundaciones }\end{array}$ & \\
\hline
\end{tabular}

\author{
Comentarios
}

Sin problemas por deslizamientos.

Se deben realizar medidas correctivas menores para asegurar la estabilidad del sector.

Potencial alto de deslizamientos, se deben implementar medidas correctivas mayores para asegurar la estabilidad del sector.

Potencial muy alto de deslizamientos, se deben

implementar medidas correctivas mayores para asegurar la estabilidad del sector.

Posibilidad de ocurrencia de flujos y avalanchas de lodo y rocas, disparadas por deslizamientos en la sección superior de la ladera.

Posibilidad de conjugación de eventos de flujos de lodo y rocas e inundaciones.

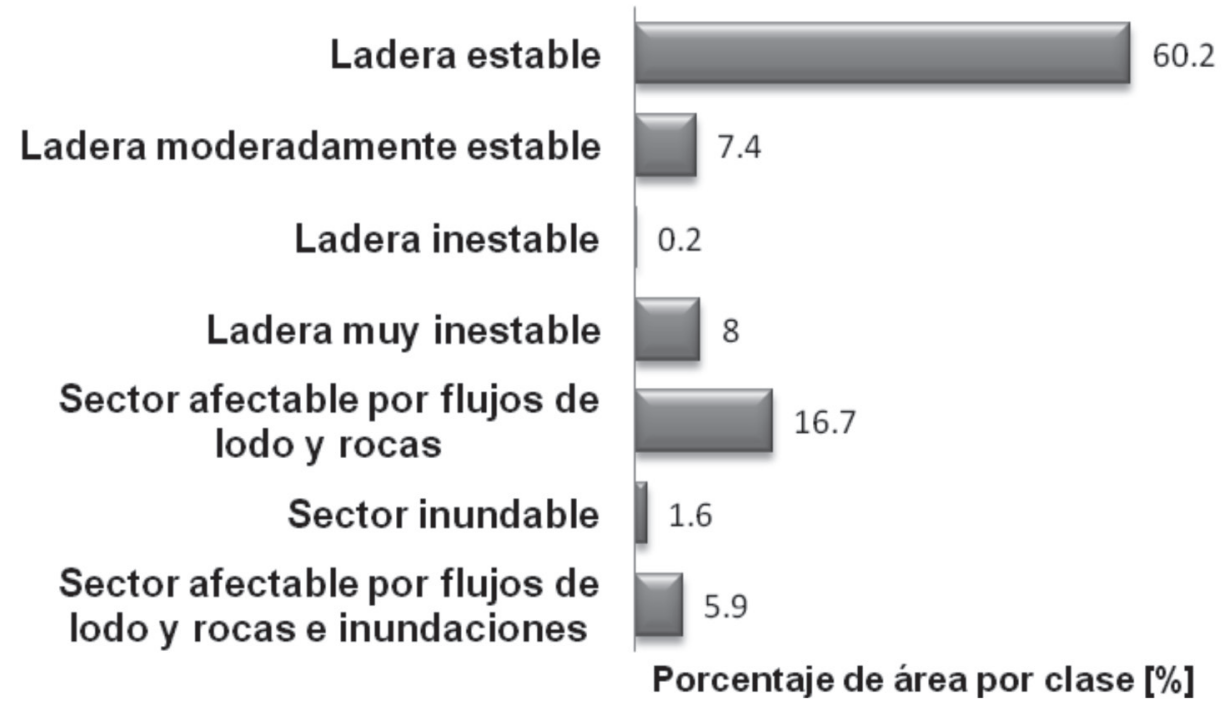

Figura 6. Histograma de porcentajes de área por clase del mapa de estabilidad en condiciones estáticas, Bajo Los Anonos, Escazú, San José.

Fuente: (El autor). 


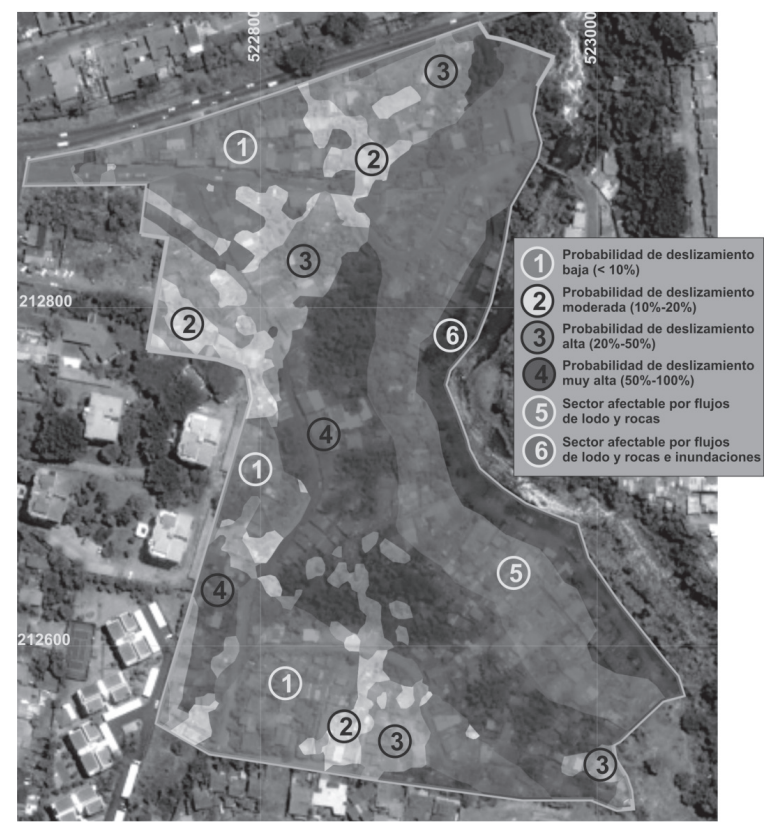

Figura 7. Mapa de estabilidad en condiciones pseudoestáticas y procesos geomorfológicos conexos, Bajo Los Anonos, Escazú, San José.

Fuente: (El autor).

estabilidad en condiciones estáticas, referidas al área cubierta por cada clase con respecto al área total del sitio de estudio. El área total estudiada en el Bajo Los Anonos es de $91667,42 \mathrm{~m}^{2}$, es decir, aproximadamente 9,2 ha.

De la Figura 6 se establece que en condiciones estáticas, el 60,2\% del área estudia comprende laderas estables. El 7,4\% requiere medidas correctivas menores para asegurar su estabilidad. Por otro lado, el 8,2 \% del área presenta condiciones de inestabilidad y el 24,2 $\%$ puede ser afectado por flujos de lodo y rocas, inundaciones o la conjugación de ambas.

\subsection{Resultados del análisis de estabilidad en condiciones pseudoestáticas}

Este análisis se realiza calculando la probabilidad de ocurrencia de deslizamientos para cada pixel del mapa y reclasificando los resultados de acuerdo con el Cuadro 3. El mapa final (Figura 7) incluye la incorporación de procesos relacionados con la ocurrencia de flujos de lodo y rocas, así como de inundaciones.
En la Figura 8 se presenta la distribución porcentual de las diferentes clases del mapa de estabilidad en condiciones estáticas, referidas al área cubierta por cada clase con respecto al área total del sitio de estudio. De la Figura 7 se establece que en condiciones pseudoestáticas, el $16,1 \%$ del área estudia comprende laderas con probabilidad baja de ocurrencia de deslizamientos. El 8,4\% representa una probabilidad moderada. Por otro lado, el 40,6\% del área cuenta con una probabilidad de alta a muy alta y el $43,6 \%$ puede ser afectado por flujos de lodo y rocas, inundaciones o la conjugación de ambas.

\section{CONCLUSIONES}

1. Las laderas del Bajo Los Anonos están constituidas por rocas bastante competentes (ignimbritas) y depósitos volcánicos (lahares y cenizas) de características mecánicas relativamente deficientes. La parte plana, cercana al río, es una terraza fluvial del río Tiribí.

2. El Bajo Los Anonos está localizado parcialmente en el cañón fluvial del río 


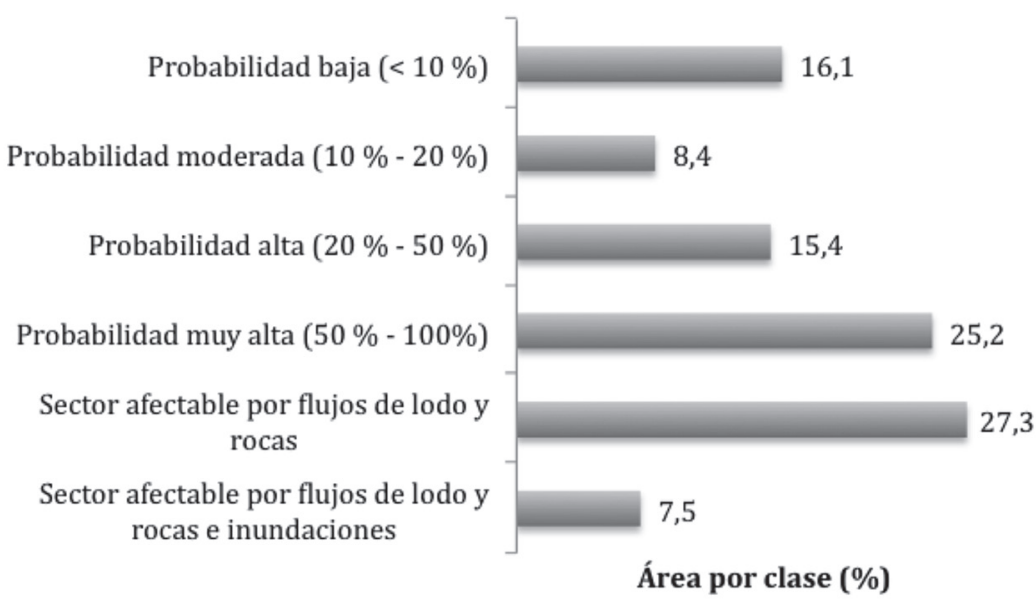

Figura 8. Histograma de porcentajes de área por clase del mapa de estabilidad en condiciones pseudoestáticas, Bajo Los Anonos, Escazú, San José.

Fuente: (El autor).

Tiribí y en campos de lahares y cenizas de pendientes moderadas a bajas.

3. Los suelos volcánicos de la parte superior de la ladera presentan la mayor problemática desde el punto de vista de los deslizamientos, pues son capaces de desarrollar eventos compuestos de deslizamientos y flujos, en caso de actividad sísmica, los cuales se moverían hacia la terraza fluvial localizada en la parte baja.

4. En condiciones estáticas, es decir sin considerar actividad sísmica, el 60,2\% del área estudiada comprende laderas estables, el $7,4 \%$ requiere medidas correctivas menores para asegurar su estabilidad, el 8,2\% presenta condiciones de inestabilidad y el $24,2 \%$ puede ser afectado por flujos de lodo y rocas, inundaciones o la conjugación de ambas.

5. En condiciones pseudoestáticas, es decir considerando el efecto de la actividad sísmica, el 16,1 \% del área estudiada comprende laderas con probabilidad baja de ocurrencia de deslizamientos, el 8,4\% representa una probabilidad moderada, el 40,6\% del área cuenta con una probabilidad de alta a muy alta y el 43,6\% puede ser afectado por flujos de lodo y rocas, inundaciones o la conjugación de ambas.

6. En términos generales, se puede concluir que poco más de $1 / 6$ del área del Bajo Los
Anonos se puede considerar segura para la construcción de infraestructura habitacional.

\section{REFERENCIAS BIBLIOGRÁFICAS}

Haneberg, W. (2005). PISA: Probabilistic infinite slope analysis User Manual. (Version 1.0): CA, EU: Haneberg Geo-science.

Mora, R. (1999). Capacidad de soporte de suelos residuales y estabilidad de taludes en roca, formación depósitos de avalancha ardiente, Tajo COMAG, Pavas, San José, Costa Rica. Empresa Auxiliar 04, Escuela Centroamericana de Geología, Universidad de Costa Rica. Informe inédito.

\section{SOBRE EL AUTOR}

\section{Rolando Mora Chinchilla}

Ingeniero Geólogo,

Profesor de la Escuela Centroamericana de Geología,

Universidad de Costa Rica

Teléfono: (506) 2511-4236

Correo electrónico: rmorach@geologia.ucr. ac.cr 
